\title{
Frontières
}

\section{Vivre avec la mort en tête}

\section{Le rapport des personnes âgées à la vieillesse, à la mort et aux médicaments}

\section{Johanne Collin}

Volume 16, numéro 1, automne 2003

Remède ou poison?

URI : https://id.erudit.org/iderudit/1073763ar

DOI : https://doi.org/10.7202/1073763ar

Aller au sommaire du numéro

Éditeur(s)

Université du Québec à Montréal

ISSN

1180-3479 (imprimé)

1916-0976 (numérique)

Découvrir la revue

Citer cet article

Collin, J. (2003). Vivre avec la mort en tête : le rapport des personnes âgées à la vieillesse, à la mort et aux médicaments. Frontières, 16(1), 64-70.

https://doi.org/10.7202/1073763ar
Résumé de l'article

Cet article propose une analyse du médicament comme révélateur de notre rapport au corps et au temps en contexte de modernité avancée. Il offre un nouveau regard sur la problématique de la consommation de médicaments chez les personnes âgées en déplaçant la focale, du discours médical et de santé publique (surconsommation, prescription inadéquate, inobservance, etc.) vers celui, plus intérieur, concernant la façon dont les personnes âgées vivent leur vieillissement et la perspective d'une mort proche. Il contribue ainsi à une réflexion plus large et en construction sur le médicament comme objet social et culturel dans nos sociétés occidentales avancées. 


\section{Résumé}

Cet article propose une analyse du médicament comme révélateur de notre rapport au corps et au temps en contexte de modernité avancée. II offre un nouveau regard sur la problématique de la consommation de médicaments chez les personnes âgées en déplaçant la focale, du discours médical et de santé publique (surconsommation, prescription inadéquate, inobservance, etc.) vers celui, plus intérieur, concernant la façon dont les personnes âgées vivent leur vieillissement et la perspective d'une mort proche. II contribue ainsi à une réflexion plus large et en construction sur le médicament comme objet social et culturel dans nos sociétés occidentales avancées.

Mots clés : médicament-personne âgée - corps - vieillissement.

\begin{abstract}
This paper proposes an analysis of medication as revealing our relationship with the body and with time in a context of advanced modernity. It proposes a new look at the issue of the consumption of medications in the elderly by displacing the lens from medical discourse and public health (over-consumption, inadequate prescriptions, adherence, etc.) to another, more intimate perspective, by considering the ways that the elderly live their old age and the prospect of an imminent death. It contributes, in this respect, to the larger contemporary debate on medication as a social and cultural object in our advanced Western societies.
\end{abstract}

Key words: medication - elderly - body aging.

\section{VIVRE \\ AVEC LA MORT EN TÊTE Le rapport des personnes âgées à la vieillesse, à la mort et aux médicaments}

\author{
Johanne Collin, Ph.D. \\ professeure, Faculté de pharmacie, \\ Université de Montréal.
}

Cet article a pour objectif de mettre au jour une analyse du médicament comme révélateur de notre rapport au corps et au temps en contexte de modernité avancée. Dans nos sociétés, les personnes âgées, de plus en plus nombreuses, tissent pour la plupart un lien étroit avec le médicament; lien dont la portée dépasse largement la dimension instrumentale et strictement biomédicale. Objet social et culturel, le médicament recèle alors une portée symbolique forte en ce qu'il constitue un rempart contre la détérioration du corps, la douleur, la souffrance psychique et la mort. Le rapport à la mort se vit en termes de temporalité et transite forcément par une réflexion sur la vieillesse, moment où la mort se fait plus tangible et plus proche.

Il s'agit dès lors ici de proposer un nouveau regard sur la problématique de la consommation de médicaments chez les personnes âgées en déplaçant la focale, du discours médical et de santé publique (surconsommation, prescription inadéquate, inobservance, etc.) vers celui, plus intérieur, concernant la façon dont les personnes âgées vivent leur vieillissement et la pers- pective d'une mort proche. Le médicament s'y présente alors sous une autre forme. Le vieillissement et l'entrée dans la «vieillesse », tels que révélés par le discours des personnes âgées, nous interpellent également fortement sur les deux vecteurs majeurs de la matrice sociale que constituent le rapport au temps et au corps en contexte de modernité avancée. Il s'agit dès lors, à terme, de contribuer à travers la focalisation sur la vieillesse et la mort à une réflexion plus large et en construction sur le médicament comme objet social et culturel dans nos sociétés occidentales avancées.

\section{LA PROBLÉMATIQUE DU RAPPORT AU MÉDICAMENT CHEZ LES PERSONNES ÂGÉES}

Les personnes âgées sont souvent atteintes de pathologies multiples et chroniques. Il n'est pas rare qu'elles aient, dans ce contexte, à se soumettre à une pharmacothérapie complexe où plusieurs médicaments entrent en jeu, multipliant du même coup les risques de chevauchements thérapeutiques, de dosages trop élevés ou d'interactions médicamenteuses nuisibles. De fait, une étude menée au début des années 1990 au Québec révélait que 45,6 \% des personnes âgées consommant des médicaments avaient à leur 
dossier au moins une ordonnance potentiellement non appropriée (Tamblyn et al., 1994).

Dans les écrits, l'utilisation non appropriée des médicaments est mentionnée à partir du début des années 1990 comme l'un des déterminants importants de la perte d'autonomie et de l'utilisation croissante des services de santé par les personnes âgées, le recours inadéquat étant d'emblée identifié comme responsable de plus de la moitié des hospitalisations de personnes âgées causées par les médicaments (Tamblyn, 1996, 1997). Dans la foulée du questionnement soulevé par ces recherches, les médicaments psychotropes s'avéraient fréquemment mis en cause. Il est vrai que la consommation de psychotropes, concernant de 20 à $30 \%$ des personnes âgées vivant dans la communauté, était considérée comme particulièrement préoccupante compte tenu des effets qui lui sont potentiellement associés (troubles cognitifs, psychomoteurs, de dépendance, etc.) (Cohen et Collin, 2000 ; Collin, 2001).

La consommation inadéquate, la surconsommation ou sous-consommation relevée dans l'analyse du rapport des personnes âgées au médicament a dès lors orienté une grande partie de la recherche causale vers les déterminants de la prescription. Les études sur cette question se sont multipliées, faisaient état de trois types de déterminants : ceux qui concernent les caractéristiques des médecins prescripteurs (Beall et al., 1996; Monette et al., 1994), ceux relevant du contexte de leur pratique (Davidson et al., 1994) et finalement, ceux liés à l'interaction entre patients et médecins lors de la consultation (Di Matteo, 1993).

Toutefois, explorer la problématique de la consommation inadéquate ne pouvait se faire sans mettre en cause également le comportement des consommateurs et les déterminants de leur recours à la médication. En parallèle proliféraient alors les études sur la question de l'inobservance au médicament (Claesson et al., 1999 ; Coambs et al., 1995). Les écrits sur cette question concernant les personnes âgées introduisaient une distinction entre inobservance volontaire et involontaire. Si, dans le premier cas, le patient était considéré comme refusant délibérément de suivre les recommandations médicales, le second cas de figure était d'emblée considéré comme le plus répandu. La personne âgée non observante était appréhendée comme la victime passive d'une situation thérapeutique qui la dépasse, parce que trop complexe; le manque d'informations ou de connaissances, ou encore, les problèmes cognitifs (confusion et perte de mémoire) étant invoqués au premier chef comme responsables d'un recours inadéquat aux médicaments prescrits (Blenkiron, 1996).
Si les études épidémiologiques sur le rapport des personnes âgées au médicament se sont multipliées, peu d'entre elles ont cherché à l'explorer sous l'angle de la subjectivité, à travers le discours des patients âgés, leurs représentations du « temps » et du «corps» par lesquelles se construit ce rapport au médicament.

\section{LE RAPPORT AU TEMPS ET AU CORPS EN CONTEXTE DE MODERNITÉ AVANCÉE}

Les personnes âgées se situent à contrecourant des valeurs axées sur le rendement, la performance, le dynamisme, la vitesse, l'immédiateté qui caractérisent la modernité avancée. Notre époque serait en effet caractérisée par une propension au recentrage sur le présent et au « surinvestissement narcissique de l'espace temporel » (Corin, 1985). L'individu aurait perdu foi, globalement, dans le politique pour changer la société et nourrirait une indifférence définitive envers le passé et l'avenir. Il fonctionnerait, par contre, en parfaite concordance avec une société de consommation valorisant le confort individuel physique et psychologique sur lequel repose le narcissisme hédoniste ambiant (Lipovetsky, 1983). entre sphère publique et sphère privée. Plus globalement, le passage d'une société disciplinaire, où les normes sociales étaient clairement établies et soutenues par les institutions (l'Église, l'État providence, la famille traditionnelle), à une société permissive où les mécanismes disciplinaires, de normalisation et de standardisation des besoins se sont considérablement affaiblis, serait en cause, avec comme conséquence le relâchement de la pression disciplinaire et la multiplication des repères au niveau des normes de fonctionnement et des valeurs. Cette multiplication des repères conduirait en définitive à une perte de repères, à travers l'élargissement des possibles, ainsi qu'à l'accroissement de l'incertitude et de la pression psychique sur les individus eux-mêmes. "Majorer son autonomie individuelle, accroître son potentiel de développement personnel, améliorer son apparence physique: toutes ces nouvelles normes poussent à accentuer les contrôles que l'individu doit exercer sur lui-même pour avoir une vie sociale, professionnelle, affective»(Ehrenberg, 1992, p. 63).

La modernité avancée engage donc un rapport au temps et au corps bien particulier. Tout se passe comme s'il y avait déplacement des dispositifs de régulation des

\section{LA SOLITUDE, QUI SOUVENT ACCOMPAGNE LA VIEILLESSE,}

INCARNE EN OUTRE LE TEMPS SUSPENDU DANS UNE SOCIÉTÉ QUI VALORISE LE MOUVEMENT.

Des transformations structurelles seraient à l'origine de ces valeurs et de cette nouvelle normativité. Un premier constat concerne la déstructuration des liens sociaux (Lipovetsky, 1983; Ehrenberg, 1998). Par là, on entend le démantèlement des liens familiaux mais également des liens collectifs (politiques, syndicaux, d'entraide) qui soutenaient les individus dans leurs rapports à la sphère publique. Depuis quelques décennies, les modes d'action de la politique et des institutions feraient en effet de plus en plus appel à la responsabilité individuelle, les normes de conduite en société exerçant en ce sens de fortes pressions sur l'individu pour qu'il se prenne en charge (Ehrenberg, 1998). Il se serait donc produit un report sur l'individu des responsabilités auparavant assumées, ou à tout le moins médiatisées, par les institutions, les cadres communautaires et les mouvements collectifs.

Cette désintégration des liens collectifs et l'individualisation qui caractérise la modernité avancée s'accompagneraient dès lors d'un brouillage des frontières institutions productrices de normes concernant notamment les valeurs morales telles la famille, l'Église, etc., vers d'autres institutions centrées sur la santé, le corps, l'apparence, la productivité, la consommation, bref, une matérialité porteuse de certitudes dans une société où les repères se sont multipliés et où le tracé des frontières et des limites n'est plus très clair. La nouvelle norme de santé et d'apparence qui caractérise nos sociétés occidentales aurait donc pour effet de tronquer la perspective temporelle et de faire en sorte que les individus s'inscrivent dans des interactions ponctuelles plutôt que dans des rôles sociaux ancrés sur une temporalité longue.

D'où la mise en scène du corps comme marqueur de notre identité. À ce stade où nous sommes de "l'avancée individualiste », " $[\ldots]$ le corps devient le refuge et la valeur ultime, ce qui reste quand les autres se font évanescents et que toute relation sociale se fait précaire. Le corps demeure l'ancre, seule susceptible de river le sujet à une certitude, encore provisoire certes, mais par laquelle il peut se rattacher à une 
sensibilité commune, rencontrer les autres, participer au flux des signes et se sentir toujours en prise sur une société où règne l'incertitude» (Le Breton, 1990, p. 159). En effet, " En se retirant partiellement des anciennes solidarités sociales, en assumant une certaine atomisation de sa condition, l'individu est invité à découvrir son corps comme une forme disponible à son action ou à sa découverte, un espace dont il convient d'entretenir la séduction, d'explorer plus avant les limites. Le corps est le tenant lieu de l'individu, son partenaire. C'est bien la perte de la chair du monde qui force l'acteur à se pencher sur son corps pour donner chair à son existence » (Le Breton, 2002, p. 110).

\section{LA VIEILLESSE : \\ À CONTRE-COURANT \\ DU RAPPORT AU TEMPS \\ ET AU CORPS QUI CARACTÉRISE \\ LA MODERNITÉ}

Un rapport au temps ancré sur le présent et un rapport au corps visant l'ultime de l'apparence et du confort ne sont, dès lors, guère propices à la valorisation de ce qu'incarne le vieillissement. Ce dernier renvoie plutôt au «temps métabolisé » (Le Breton, 1990), c'est-à-dire à l'idée de la progression linéaire et irrémédiable vers une détérioration de ce repère majeur que constitue le corps, vers une défection progressive de ses capacités, vers un environnement socioaffectif de plus en plus exsangue. La solitude, qui souvent accompagne la vieillesse, incarne en outre le temps suspendu dans une société qui valorise le mouvement.

Cette marginalité imposée, et fortement intériorisée par les personnes âgées, nous renvoie au concept de stigmate (Goffman, 1975), que des auteurs ont métaphorisé dans l'expression «mask of old age» (Conway et Hockey, 1998). Il y a non seulement stigmate, mais distanciation entre la présentation de soi et son rôle dans la vie de tous les jours. Le corps est bien celui qui trahit l'âge qu'à un certain moment on ne peut plus camoufler. C'est bien le corps, et les signes de vieillissement qui l'envahissent progressivement, qui entraînent cette distance entre soi et l'image de soi comme vieux.

De fait, l'univers discursif des personnes âgées témoigne de l'intériorisation d'une image déficitaire de la vieillesse. Interviewées dans le cadre d'une de nos enquêtes $^{1}$, celles-ci nous livrent un discours explicite à cet égard à travers le regard qu'elles portent sur leur corps vieux:

D'un coup viennent les p'tits bobos, puis toutes sortes de choses. [...] Puis, je regarde vieillir mon mari, et je dis

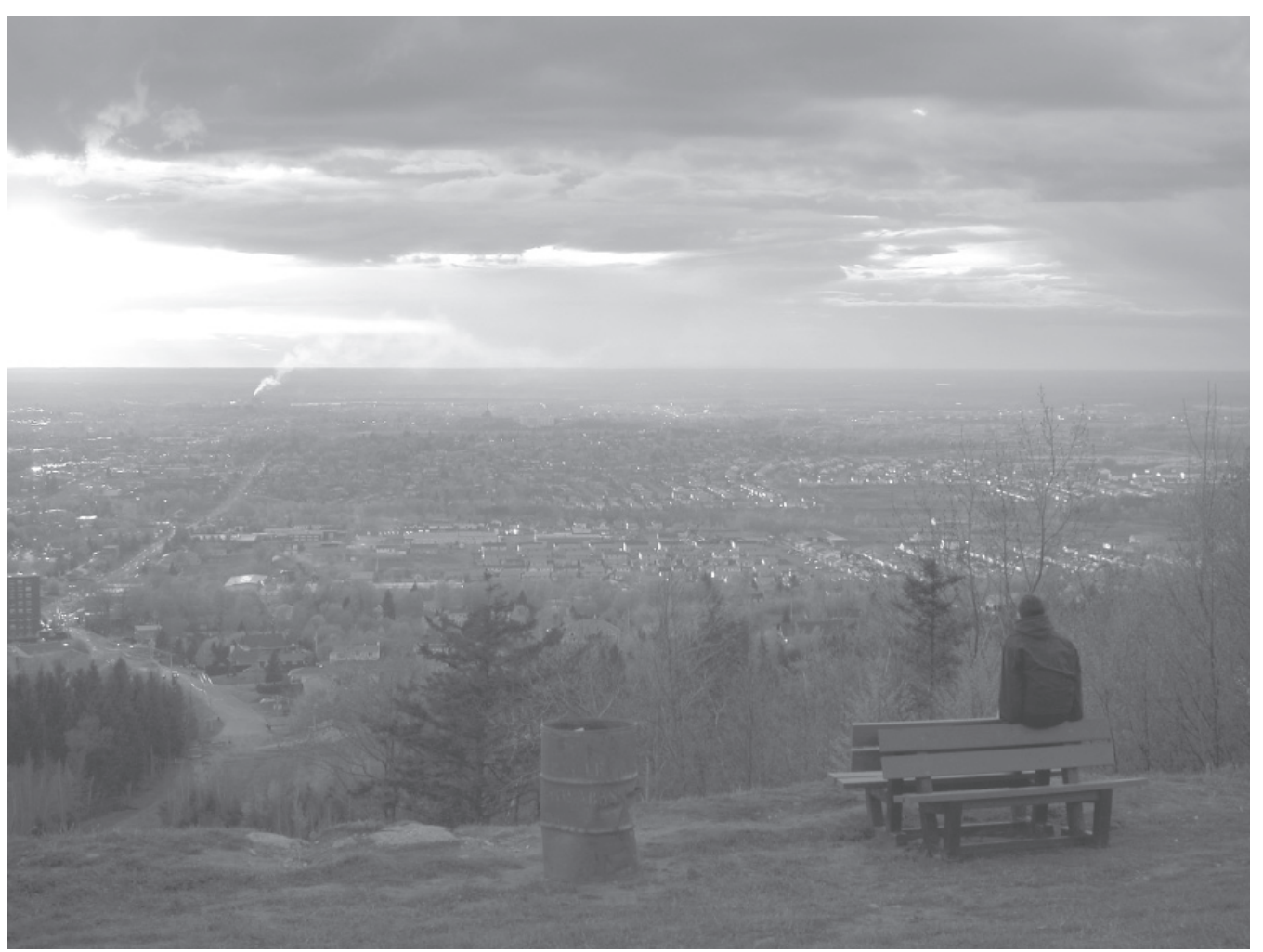

André Clément, Mont St-Michel

«ah! mon Dieu» [...] Puis lui doit faire la même chose, me regarder, t'sais. «Elle a vieilli la vieille» [rires]... D'un autre côté, en tant qu'homme debout, on sauve les apparences. Tout l'monde nous pense en parfaite santé, puis c'est tant mieux, t'sais. Le fait de juste marcher comme tout l'monde, ça éblouit. Tandis qu'ici, là, les cannes, les marchettes... là, ça paraît plus vieux.

L'image de soi comme vieux se pose d'abord sur le visage, ce repère identitaire majeur, si éloquemment porteur de cette distanciation entre le corps et soi. Le visage est en effet:

\section{[...] de toutes les zones du corps humain, celle où se condensent les valeurs les plus élevées. [...] L'altéra- tion du visage qui montre une trace de lésion aux yeux des autres est vécue comme un drame, à l'image parfois d'une privation d'identité. Une bles- sure, même grave, et laissant une cicatrice profonde à un bras, à une jambe ou sur le ventre n'enlaidit pas ; elle ne modifie en rien le sentiment d'identité. Le visage est, avec le sexe, le lieu le plus investi, le plus solidaire du Moi (Le Breton, 2002, p. 89).}

S'adressant à l'intervieweuse, c'est ce qu'exprime l'une des participantes: "Ben, regarde-moi pas là, je suis pleine de rides. Hé que j'hais ça vieillir! Ah, j'hais ça. Y a pas personne qui aime ça. » Pour une autre : «si je pouvais trouver des pilules pour me faire rajeunir $[\ldots]$ je la prendrais tout de suite là $[\ldots] \mathrm{Y}$ en a pas $[\ldots]$ pis on a pas trouvé de fontaine. Faut que j'accepte [...] faut que je m'accepte dans le miroir, comme je suis $[\ldots] »$.

Le corps vieux est un marqueur d'identité sociale, d'une image déficitaire d'autant mieux intériorisée que ce corps, manifestant déjà ses limites de façon récurrente, devient un corps-prison lorsqu'il est atteint de maladies chroniques. C'est par le détail que les participants à l'étude décrivent l'effet du temps sur leur corps:

Plus tu vieillis, plus tes artères durcissent $[\ldots]$ elles croutonnent $[\ldots]$ [en vieillissant] nos os, pis nos muscles épaississent. C'est pour ça qu'on vient qu'on a plus de taille, et puis nos muscles du dos nous lâchent fait que [...] c'est pour ça qu'on fait un peu d'abdomen [...] [on est] plus capable de se retenir [...] Quand on est jeune, on a l'énergie de se tenir, mais là on dirait que $[\ldots]$ tout s'affaisse $[\ldots]$ Notre peau, ça ne revient plus, on n'a plus d'élasticité. Et puis, c'est normal en vieillissant qu'on ait des douleurs comme ça. Sont rares, rares, rares les personnes qui ont pas des douleurs. Mais qu'est-ce que tu veux, on appelle ça l'«âge d'or », moi j'appelle ça [...] l'âge des vicissitudes [rires].

Dans cette longue énumération du travail du temps sur le corps est également 
évoquée, au-delà du paraître, l'atteinte plus basique encore à l'être et à l'action :

En vieillissant [...] aussitôt qu'on se fatigue, ben, ça pense moins vite là, là. $\mathrm{Y}$ a moins de concentration [...] Puis c'est notre mémoire qui s'embrouille aussi en vieillissant. Y en a qui gardent leur mémoire, j'ai des amis, moi, ils ont une mémoire fabuleuse. Mais moi, j'en ai pas, c'est effrayant. Quand on vieillit, on est plus anxieux. Graduellement, il y a certaines choses que t'es plus capable faire. Ça, ça te fait perdre [...] des fois je perds patience.

Dans le rapport au corps, d'abord par la surface - les apparences, les rides et tous les signes à fleur de peau du processus interne d'usure et de changement -, ce que l'on croyait presque immuable commence à nous échapper. L'apparence témoigne de ce que l'on aurait voulu cacher, de la faiblesse et du déficit vers lequel on s'achemine. Mais la vieillesse, c'est aussi le corps qui nous lâche à travers la perte de contrôle de ses sens (la vue et l'ouïe surtout), la perte de l'équilibre, de la capacité à se mouvoir seul, sans prothèse et sans aide. L'altération, en somme, du rapport à son environnement:

J'peux pas toujours être comme ça, là, avoir d'la misère à monter, j'camoufle un peu mais [...] Et vous savez que maintenant, plus j'vais, moins je suis capable de prendre un bain. Plus capable de me retourner dans le bain. La qualité de vie s'en trouve [...] Ceux qui peuvent se payer une infirmière vingt-quatre heures par jour, ça peut aller, mais quand [...] t'as pas les moyens, t'es pas [...] qu'est-ce qui va arriver, là, maintenant?

Les pertes sensorielles, quant à elles, confirment la perte de contrôle de leur corps ; elles confirment également qu'on ne peut échapper à la vieillesse parce qu'elles sont sans retour et qu'elles témoignent de ce rétrécissement des plaisirs et du rapport au monde.

Cette transformation qui, dans la plupart des cas, s'avère sans perspective d'amélioration, rend les espoirs modestes : « si jamais je viens qu'à pouvoir marcher comme $\mathrm{y}$ faut, je vais être ben heureux». Parce qu'en somme, la tension vers l'action, la capacité à être actif est synonyme de «non-vieillissement » : «Les [...] les gens âgés, ça devrait pas arrêter de travailler [...] là, ils sont là, ils s'assoient là, puis regardent la télé [...] La télé, c'est une peste pour la santé des gens. » Pour un autre répondant: "À dire, je me sens pas vieux. Je me sens jeune. Je remercie [...] parce que quand je vois le monde, surtout ici, y en a plusieurs qui sont handicapés [...] [tandis que nous] on est presque sortis à tous les jours ».

En fait, la vieillesse partage plusieurs caractéristiques avec la maladie chronique. Dans un cas comme dans l'autre, il y a rupture biographique. Tout comme celle du malade chronique, l'identité de la personne qui entre dans la vieillesse, entrée symbolisée socialement par le retrait de la vie active, est reliée au temps d'une manière singulière: aucun retour à une identité antérieure n'est pensable (Bury, 1982; Corbin et Strauss, 1987). Et ce, d'autant plus que face à l'angoisse du processus de détérioration auquel est confrontée la personne âgée, la société ne lui fournit plus «les repères qui lui permettent de penser son maintien et sa continuité symbolique, par delà sa disparition physique: que ce soit à travers sa position dans une suite de générations ou en référence à une certaine conception de l'au-delà » (Corin, 1985, p. 477).

Les activités sociales et les relations avec les proches sont également profondément modifiées. Une participante nouvellement installée dans un complexe domiciliaire pour personnes âgées se rebiffe contre sa nouvelle image identitaire: "Bon, ici, on est toutes des p'tites madames [...] Ça j'haïs ça. Moi, j'ai travaillé dans des résidences pour personnes âgées, puis [...] je trouve que, c'est pas parce qu'on vieillit que tout le monde doit [...] nous parler comme si on n'était pas capable de raisonner, là. »

À l'instar de l'entrée dans la maladie chronique également, le retrait de la vie active implique de repenser l'organisation des structures qui encadrent le rapport social et instrumental au quotidien et de tendre vers l'établissement d'une nouvelle normalité (Charmaz, 2000). Comme le déplore l'un des participants: «Y a des vieux ici qui sont pris, hein! Ils sont seuls. Ils ont de la famille qui viennent même pas les voir. Quand ils viennent, c'est [...] pour de l'argent! Ben, c'est ça aujourd'hui [...]» Plusieurs souffrent particulièrement de leur isolement : « Rendu à mon âge, là, j'intéresse plus personne. Si j'étais actif comme j'étais ben c'est entendu [...] mais rendu à l'âge que j'ai là $[. .$.$] » D'autres se rebiffent contre$ la façon dont ils se sentent mis à l'écart en tant que personnes âgées : «Les jeunes [...] c'est écœurant! Y aiment pas les personnes âgées, fait que, dès qu'on vieillit, on ferme notre boîte pis on parle plus. » Et encore :

Ce qui arrive actuellement et qui me choque un peu [...] c'est que, nous, les personnes âgées, là, ou en voie de devenir âgées, on nous a traitées, là, on va peut-être le faire un peu moins, parce qu'on coûte cher. Alors, on nous a traitées [...] on nous a bien donné tout ça, pour nous faire vieillir [...] Hein! Nous garder en vie [...] mais [...] dans quelles conditions?

\section{VIVRE AVEC LA MORT EN TÊTE :} VIEILLESSE ET RAPPORT AU TEMPS

Si la modernité avancée valorise l'immédiateté, dans un rapport au temps qui oscille « entre le fugitif et l'éternel» (Baudelaire), le «temps» des personnes âgées est tout aussi obsessif mais se décline de multiples façons: temps nié, temps bloqué, temps anticipé et temps fin de parcours.

Le temps bloqué, c'est-à-dire sans possibilité de projection dans l'avenir, s'exprime ainsi par les remarques de deux participants: «[...] quand t'as vingt ans, oui, quand t'as trente ans, oui [...] trente-cinq ans, oui [...] Des projets à très long terme [...] Des projets à long terme, finir ta résidence, ta maison [...] payer ta voiture, payer çi, payer ça [...] mais rendu à mon âge, c'est terminé ça.» Et encore : «Fait que ça, c'est la dernière étape de ma vie, pis on attend le dénouement. On s'en va par là, au grand dénouement mais quand viendra-t-il? Lequel partira le premier, on le sait pas. »

Du discours des personnes âgées peuvent cependant émerger des perspectives plus positives où domine le temps nié à travers la focalisation sur l'instant présent. Lorsqu'on lui demande comment elle vit le vieillissement, une répondante affirme : «Je m'occupe tout le temps. Tout le temps, tout le temps. Je suis toujours occupée, je suis toujours pressée. J'ai toujours quelque chose à faire. Puis je sors .» Pour un autre participant à l'enquête :

Ce n'est pas difficile de vieillir, parce que d'abord, tous les matins sans exception, je dis à Dieu : "Merci mon Dieu, tu me donnes une autre journée, c'est très gentil de ta part. Merci beaucoup.»Quand tu es prêt à vieillir et deuxièmement quand tu acceptes volontairement, sans effort, de vieillir, tu ne vieillis presque pas, t'sais.

Le temps nié devient aussi tempsrécompense pour certains :

Je vis les belles années qui me restent [...] pour moi, moi [...] j'me considère chanceux [...] Si j'avais 50 ans, là, peutêtre j'accepterais pas [...] de rien faire. Faudrait que je travaille. Avec l'âge, j'accepte ça puis [...] c'est une étape. Les étapes de la vie changent puis [...] faut que tu t'adaptes à survivre [...] Là, je me sens très bien. Très heureux en couple puis $[\ldots]$ plus de travail.

Cependant, ces perspectives temporelles courtes en côtoient d'autres qui se fondent sur une projection dans l'avenir, si angoissant 
que celui-ci puisse être. Le temps anticipé s'établit dès lors sur une gestion de l'incertitude et de la peur de l'avenir. Craignant ainsi - sans raison objective - d'être un jour atteinte de la maladie d'Alzheimer, une participante affirme : "J'espère que si j'en fais, j'aurai pas conscience que je suis comme ça. Pis j'ai dit à mon mari: "tu me placeras" $[\ldots]$ à l'âge qu'on va être rendu si on en fait $[. .$.$] , on ne serait peut-être pas$ capable d'avoir soin l'un de l'autre [...]» Cette crainte de ce qui peut survenir l'a également incitée, de concert avec son mari, à faire à l'avance tous leurs arrangements funéraires de façon à ce que les enfants n'aient pas à s'en occuper : "ils n'auront pas de troubles, c'est un acte d'amour ça ». Se félicitant d'avoir emménagé récemment dans un complexe domiciliaire pour personnes âgées, une participante nous confie : «si, moi, je meurs avant lui, je sais qu'il ne sera pas tout seul parce qu'on a un groupe d'amis ». Pour une autre: "Ça ne m'intéresse pas d'aller en résidence, mais le temps va venir si je meurs pas avant, le temps va venir. Mais, j'ai mon mari aussi. Je ne peux pas le laisser seul lui. Lui aussi, il devrait se [faire] placer. Il ne peut rien faire dans la maison $[\ldots] »$

Ce «temps anticipé» de la maladie et de la mort, où se perçoit un certain désir d'agir sur les conditions et les contextes entourant ces événements, se distingue du temps fin de parcours, qui lui, constitue en quelque sorte la «chronique d'une mort annoncée ». Chaque événement habituellement récurrent est alors vécu comme le dernier (dernier Noël, dernier anniversaire, dernier déménagement, etc.) ; sans certitude absolue certes, mais porté par le besoin d'exorciser ses peurs et peut-être aussi par le désir secret d'être rassuré. Malgré l'évocation de la mort, le ton emprunté n'est cependant jamais triste :

Je l'arrêterais le temps maintenant.

Parce que je peux pas faire

autrement [...] Je pense souvent [...]

C'est pas pathétique, mais que ce

n'est peut-être pas pour longtemps [...] que le Noël que je prépare [...] Moi, je me rendais pas compte à quarante, cinquante, même soixante que je

mourrais [...] moi, je pensais jamais à ça.

Planifiant un voyage chez une de ses amies, une répondante rapporte : «Ben, elle m'a dit: "fais tes affaires, tu viendras à l'automne". J'y ai dit: "si je ne suis pas morte!" [rires]». Relatant enfin son dernier déménagement, une autre répondante raconte :

J'étais pas âgée âgée, mais j'étais pas jeune non plus. 73 ans, là, pour un déménagement. C'est quelque chose!
Là, je veux plus recommencer. À la madame en bas qui nous avait loué, à l'accueil, j'ai dit: "je vais sortir les deux pieds en avant, pis je laisse tout le ménage en haut [rires] ». Puis j'ai dit: "je vais m'en aller en Cadillac à Côte-des-Neiges. Ça, ça va être mon déménagement, mon dernier ».

\section{MÉDICAMENT ET VIEILLESSE EN CONTEXTE DE MODERNITÉ AVANCÉE}

Dans cette phase de l'existence où le temps - à travers son travail sur le corps devient une donnée incontournable, le médicament occupe une place centrale. Il représente le rempart contre la mort, l'assurance d'un contrôle, d'une certaine prise sur l'évolution de la maladie chronique. Son recours devient alors d'autant plus incontournable pour les personnes âgées qu'il ne fait que traduire, de façon exacerbée, la préoccupation majeure que nourrit l'individu en contexte de modernité avancée pour sa corporéité, son apparence, son confort et sa santé.

Le médicament comme objet technologique préside en effet à une certaine reconfiguration de la notion de prévention, en rendant possible l'accroissement de l'indistinction entre confort et santé. Depuis quelques décennies, la définition officielle de la santé (OMS) est passée de «l'absence de maladie » à la notion de « bien-être physique et mental». Portée par la société de consommation, cette définition ouvre la porte à une nouvelle conception de la «prévention » intimement liée au médicament. En effet, l'état de «bien-être » que constitue la nouvelle norme de santé se doit d'être préservé le plus longtemps possible à travers l'atteinte d'un confort physique, moral, psychologique de tous les instants. Le médicament devient ici un outil, non seulement pour atteindre le bien-être, mais pour le maintenir; outil dont le recours est justifié tant par l'absence de la maladie que par son apparition. Le discours sur la prévention, largement repris par l'industrie pharmaceutique, s'appuie désormais et de plus en plus sur la notion de risque et sur l'évaluation précise qu'en procure l'épidémiologie. Le risque d'accident vasculaire cérébral chez les hypertendus, celui d'ostéoporose chez les femmes ménopausées, de rechute chez les dépressifs, de dysfonctions érectiles chez les consommateurs d'antidépresseurs, conduisent à l'extension du recours "préventif » au médicament.

Il faut voir que la notion de risque, en elle-même, est également au cœur des préoccupations dans nos sociétés. Le Breton (1998) a montré comment le contexte de la modernité avancée conduit, à travers la multiplication des repères et la gestion conséquente de l'incertitude, à surinvestir les dispositifs pour contrer le " risque $^{2}$ », dispositifs parmi lesquels figure en bonne place le médicament.

Pour les personnes âgées atteintes de problèmes cardiovasculaires, le risque de mort se conjugue au présent, parce que la mort s'impose soudainement comme une chose qui peut arriver, survenir n'importe quand, une hypothèse non seulement plausible mais réaliste. Plusieurs l'auront d'ailleurs déjà côtoyée en étant victimes d'infarctus et d'accidents vasculaires cérébraux. La crainte de mourir est également présente chez ceux qui sont atteints de diabète ou de problèmes reliés à la glande thyroïde. Pour l'une des participantes, les médicaments « vitaux » qu'elle prend fonctionnent ensemble, elle ne peut se permettre d'en oublier un ou de ne pas suivre la posologie établie par son médecin. Un autre considère que ses médicaments, "c'est sa vie ».

Mais la modernité avancée soutient également le développement d'un nouveau rapport à la douleur que permettent d'actualiser les progrès en matière d'analgésiques. Selon Castra, les soins palliatifs traduisent une tendance croissante de nos sociétés occidentales à la pacification de la mort :

L'intériorisation d'une perception pacifiée des corps qui s'exprime par de multiples efforts pour diminuer toute trace de douleur ou de souffrance physique, de même que le développement d'une plus grande attention portée aux émotions peuvent être mises en lien avec une nette évolution des sensibilités et une modification des seuils de perception de la violence que constitue la mort (Castra, 2003, p. 17).

La douleur serait désormais considérée non seulement comme inutile, mais nuisible. Il faut l'éliminer et l'éviter puisqu'elle empêcherait la guérison ou encore, à défaut, une fin de vie plus douce. Il en va de même de la souffrance psychique, à plus forte raison lorsqu'il s'agit de personnes âgées.

Or, le rapport à la douleur, c'est le corpsprison, qui nous pèse et dont on ne peut s'échapper, celui qui, par moment, fait appeler la mort comme libération. En effet, la perspective de vivre le restant de ses jours avec une douleur toujours présente, même si elle est d'intensité variable, et qui tendra à s'amplifier, à se répandre davantage avec le temps fait disparaître pour ceux qui la portent la perspective du temps pour soi, du temps récompense et du temps présent. "Parce que ça arrête pas la douleur, c'est, c'est [...] Ça, c'est quelque chose à vivre, mais c'est parce que j'ai soixante et seize que je le vis facilement et que ça fait long- 
temps. On peut pas toujours avoir peur à l'année longue, t'sais ».

Il en va de même de la douleur psychique, pour laquelle le recours aux médicaments psychotropes figure pour certains prescripteurs comme une obligation morale. Comme l'exprime un médecin :

On éprouve beaucoup de compassion et moi je me dis toujours une chose: à cet âge-là est-ce que je peux faire des miracles, est-ce que je peux les faire rajeunir, est-ce que je peux leur greffer des organes neufs, est-ce que je peux leur rendre leur esprit? Non. Est-ce que je peux faire en sorte qu'ils se sentent mieux, est-ce que je peux faire disparaître leur anxiété, leur angoisse? Dans un certain sens, oui ${ }^{3}$.

$$
* * *
$$

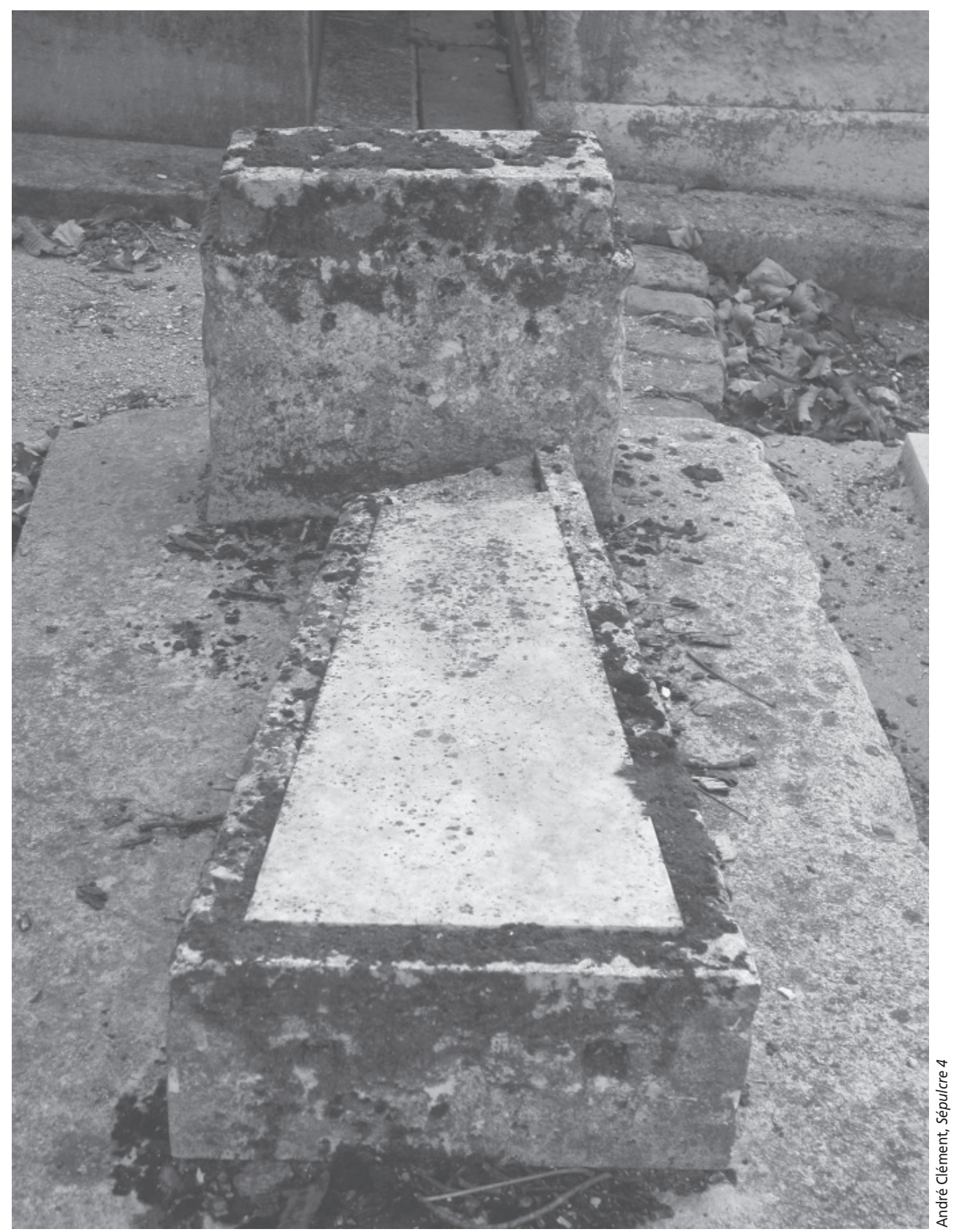

Pour les personnes âgées, le médicament est support à la souffrance en procurant temporairement l'évasion de ce corpsprison où la douleur occupe tant qu'elle empêche tout contact avec l'extérieur de soi. Mais il figure également parmi les stratégies pour échapper au stigmate de la vieillesse, pour empêcher le déséquilibre qui conduit à la maladie. Dans son extériorité, le corps vu ou agissant exprime par le recours au médicament une tension vers l'action et l'autonomie, si précieuses pour échapper à l'image accolée à la vieillesse. À travers ces fonctions qu'on lui attribue de contrôler ou contenir les problèmes de santé, soutenir le corps, réguler l'humeur, le médicament permettrait d'échapper à ce rapport au temps implacable et inflexible, ce tempssursis, ce temps-défi et ce temps-menace.
Son importance dans l'existence de la plupart des personnes âgées dépasse largement l'objet strictement technique. La perspective du vieillissement, avec tout ce qu'elle recèle de menaces pour le corps - de son apparence à son confort - et l'identité, révèle ainsi de façon exacerbée l'importance centrale du médicament comme objet social et culturel dans nos sociétés modernes avancées.

\section{Bibliographie}

BAUDELAIRE, C., 1863 / 1976, «Le peintre de la vie moderne », dans Euvres complètes, vol. II, Paris, Gallimard.

BEALL, C.S., L.A. BAUMHOVER, A.J. MAXWELL et R.E. PIERONI (1996). «Normal versus pathological aging: Knowledge of family practice residents", The Gerontologist, vol. 36, n 1, p. 113-117.

BLENKIRON, P. (1996). "The elderly and their medication: Understanding and compliance in a family practice», Postgraduate Medical Journal, $\mathrm{n}^{\circ}$ 72, p. 671-676.

BURY, M. (1982). «Chronic illness as a biographical disruption", Sociology of Health and Illness, vol. 4, $\mathrm{n}^{\circ}$ 2, p. 167-182.

CASTRA, M. (2003). Bien mourir. Sociologie des soins palliatifs, Paris, Presses universitaires de France, 365 p.

CHARMAZ, K. (2000). «Experiencing chronic illness", dans G. ALBRECHT, R. FITZPATRICK et S.C. SCRIMSHAW (dir.), Handbook of Social Studies in Health and Medicine, Londres, Thousand Oaks, New Dehli, Sage.

CLAESSON, S., A. MORRISON, I. WERTHEIMER et M. BERGER (1999). "Compliance with prescribed drugs: Challenges for the elderly population », Pharm. World Science, vol. 21, n 6, p. 256-259.

COAMBS, R.B., P. JENSEN et M. HAO HER (1995). Review of the scientific literature on the prevalence, consequences, and health costs of noncompliance and inappropriate use of prescription medication in Canada, Toronto, University of Toronto Press.

COHEN, D. et J. COLLIN (2000). La toxicomanie en lien avec la consommation de médicaments psychotropes: recension des écrits sur les personnes âgées, les femmes et les enfants, Comité de prévention des toxicomanies du Québec, ministère de la Santé et des Services sociaux, $123 \mathrm{p}$.

COLLIN, J., N. DAMESTOY et R. LALANDE (1999). «La construction d'une rationalité: les médecins face à la prescription de psychotropes aux personnes âgées ", Sciences sociales et Santé, vol. 17, n 2, p. 31-52.

COLLIN, J. (2001). «Médicaments psychotropes et personnes âgées: une socialisation de la consommation ", Revue québécoise de psychologie, vol. 22, n 2, p. 75-99.

CONWAY, S. et J. HOCKEY (1998). " Resisting the "mask" of old age?: The social meaning of lay health beliefs in later life », Ageing and Society, $\mathrm{n}^{\circ} 18$, p. 469-494. 
CORBIN, J. et A. STRAUSS (1987). «Accompaniments of chronic illness: Changes in body, self, biography, and biographical time ", Research in the Sociology of Health Care, $\mathrm{n}^{\circ} 6$, p. 249-281.

CORIN, E. (1985). « Définisseurs culturels et repères individuels : le rapport au corps chez les personnes âgées », International Journal of Psychology, $\mathrm{n}^{\circ}$ 20, p. 471-500.

DAVIDSON, W., D. MOLLOY, G. SOMERS et M. BEDARD (1994). "Relation between physician characteristics and prescribing for elderly people in New Brunswick », Canadian Medical Association Journal, vol. 150, $\mathrm{n}^{\circ}$ 6, p. 917-921.

DE BEAUVOIR, S. (1970). La vieillesse, Paris, Gallimard.

DI MATTEO, M. (1993). «Expectations in the physician-patient relationship: Implications for patient adherence to medical treatment recommendations ", dans P. BLANCK, Interpersonal Expectations: Theory, Research, and Application, Cambridge, Cambridge University Press, p. 296-315.

EHRENBERG, A. (1992). «Dépassement permanent», dans A. EHRENBERG et P. MIGNON, Drogues, politique et société, Paris, Ed. Descartes, p. 54-70.

EHRENBERG, A. (1998). La fatigue d'être soi, Paris, Odile Jacob.

GOFFMAN, E. (1975). Stigmates. Les usages sociaux des handicaps, Paris, Minuit.
LE BRETON, D. (1998). Anthropologie du corps et modernité, Paris, PUF.

LE BRETON, D. (2002). La sociologie du corps, Paris, PUF.

LIPOVETSKY, G. (1983). L'ère du vide: Essais sur l'individualisme contemporain, Paris, Gallimard.

MONETTE, J., R. TAMBLYN, P. MCLEOD, D. GAYTON et R. LAPRISE (1994). «Do medical education and practice characteristics predict inappropriate prescribing of sedativehypnotics for the elderly?", Academic Medicine, $\mathrm{n}^{\circ} 69$ (suppl.), p. 10-12.

TAMBLYN, R.M., P.J. MCLEOD, M. ABRAHAMOWICZ, J. MONETTE, D.C. GAYTON, L. BERKSON et al. (1994). "Questionable prescribing for elderly patients in Quebec», Canadian Medical Association Journal, vol. $150, \mathrm{n}^{\circ} 11$, p. 1801-1809.

TAMBLYN, R. (1996). "Medication use in seniors: Challenges and solutions », Thérapie, vol. 51, no 3, p. 269-282.

TAMBLYN, R. (1997). "Medication use in the seniors population: Optimization of physician prescribing as a means of preventing drug-related illness ", Canadian Journal on Aging (supplément), p. 147-161.

\section{Notes}

1. La place du médicament dans les stratégies de gestion de la santé et de la maladie chez les personnes âgées non institutionnalisées (Subv. Conseil québécois de la recherche sociale, 1997). Il s'agit d'une recherche qualitative menée auprès d'un échantillon de 42 personnes âgées entre 60 et 83 ans, autonomes et vivant dans la communauté. L'échantillon était composé à parts égales de personnes de milieux favorisés et défavorisés. Un entretien semi-directif d'une durée variant de 1 h 30 à 2 h 30 a été conduit à domicile auprès de chacune de ces personnes. Les entretiens ont été enregistrés, retranscrits intégralement puis codés et analysés à l'aide du logiciel Nud*ist. Pour plus d'information sur la méthodologie, voir Collin, 2001.

2. Certes, le risque exerce en même temps une attraction forte sur l'individu parce qu'il constitue une façon de se forger des repères à travers l'exploration de ses limites (Le Breton, 1996). L'individu postmoderne pris en tension entre la peur du risque et la recherche du risque "trouve son compte» avec les promesses que recèle à cet égard le médicament.

3. Cette citation est tirée d'une autre enquête que nous avons mené auprès de médecins et intitulée: La perspective des omnipraticiens face à la prescription de psychotropes aux personnes âgées (Subv. Programme de subvention en santé publique - RRSSS de Laval, 1996). Pour plus de détails sur la méthodologie et les résultats de cette enquête, voir Collin et al., 1999. 